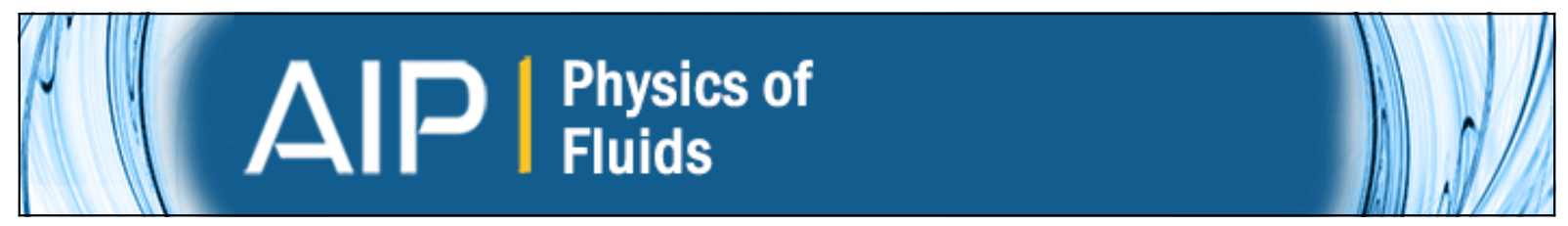

\title{
The fastest drop climbing on a wet conical fibre
}

Er Qiang Li and Sigurdur T. Thoroddsen

Citation: Physics of Fluids (1994-present) 25, 052105 (2013); doi: 10.1063/1.4805068

View online: http://dx.doi.org/10.1063/1.4805068

View Table of Contents: http://scitation.aip.org/content/aip/journal/pof2/25/5?ver=pdfcov

Published by the AIP Publishing

\section{Articles you may be interested in}

Determination of dynamic surface tension and viscosity of non-Newtonian fluids from drop oscillations Phys. Fluids 26, 113103 (2014); 10.1063/1.4901823

Damped harmonic system modeling of post-impact drop-spread dynamics on a hydrophobic surface Phys. Fluids 25, 082112 (2013); 10.1063/1.4819243

Quantitative analysis of the dripping and jetting regimes in co-flowing capillary jets

Phys. Fluids 23, 094111 (2011); 10.1063/1.3634044

Drop deformation and breakup in polystyrene/high-density polyethylene blends under oscillatory shear flow

Phys. Fluids 23, 013104 (2011); 10.1063/1.3541967

Polymer drop breakup in microchannels

Chaos 17, 041102 (2007); 10.1063/1.2786006

\section{Did your publisher get}

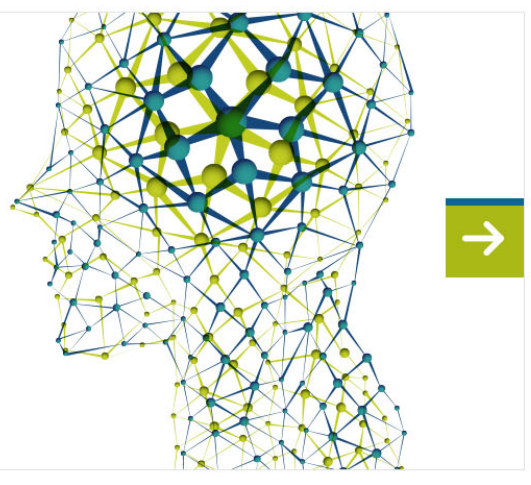




\title{
The fastest drop climbing on a wet conical fibre
}

\author{
Er Qiang $\mathrm{Li}^{1}$ and Sigurdur T. Thoroddsen ${ }^{1,2, a)}$ \\ ${ }^{1}$ Division of Physical Sciences and Engineering, King Abdullah University of Science \\ and Technology (KAUST), Thuwal 23955-6900, Saudi Arabia \\ ${ }^{2}$ Clean Combustion Research Center, King Abdullah University of Science and Technology \\ (KAUST), Thuwal 23955-6900, Saudi Arabia
}

(Received 22 December 2012; accepted 12 April 2013; published online 21 May 2013)

\begin{abstract}
We use high-speed video imaging to study the capillary-driven motion of a microdroplet along the outside of a pre-wetted conical fiber. The cones are fabricated on a glass-puller with tip diameters as small as $1 \mu \mathrm{m}$, an order of magnitude smaller than in previous studies. The liquid is fed through the hollow fiber accumulating at the fiber tip to form droplets. The droplets are initially attached to the opening as they grow in size before detaching and traveling up the cone. This detachment can produce a transient oscillation of high frequency. The spatial variation of the capillary pressure drives the droplets towards the wider side of the cone. Various liquids were used to change the surface tension by a factor of 3.5 and viscosity by a factor of 1500 . Within each droplet size and viscous-dissipation regime, the data for climbing speeds collapse on a single curve. Droplets traveling with and against gravity allow us to pinpoint the absolute strength of the driving capillary pressure and viscous stresses and thereby determine the prefactors in the dimensionless relationships. The motions are consistent with earlier results obtained from much larger cones. Translation velocities up to $270 \mathrm{~mm} / \mathrm{s}$ were observed and overall the velocities follow capillary-viscous scaling, whereas the speed of the fastest droplets is limited by inertia following their emergence at the cone tip. ( 2013 AIP Publishing LLC. [http://dx.doi.org/10.1063/1.4805068]
\end{abstract}

\section{INTRODUCTION}

The directional movement of millimeter- and submillimeter-sized droplets is omnipresent in nature. For example, desert beetles capture water from humid air through the micrometer-sized patterns of hydrophobic and hydrophilic regions on their backs; ${ }^{1}$ the Australian Moloch horridus lizard draws water through capillaries that extend from its skin to mouth, ${ }^{2}$ and the spider silk is also capable of collecting water from the humid air. ${ }^{3}$ In all these cases the directional movements of droplets occur as a result of capillary effect and are illuminating for potential applications in microfluidics, ${ }^{4}$ gas-liquid separation, ${ }^{3,5}$ surface coatings, ${ }^{6}$ and other fields involving surface chemistry and interface forces.

Capillary forces are the products of a characteristic length $d$ and surface tension $\sigma$. Thus, a directional movement can be induced once a droplet experiences a gradient of either $d^{7-9}$ or $\sigma .^{10,11}$ The latter one is well known as Marangoni flow, which is driven by an imbalance of surface tension forces resulting from gradients in temperature,,${ }^{12,13}$ electric field, ${ }^{14}$ or chemical composition. ${ }^{15-17}$ The effects of variable $d$ are best revealed in the canonical work of Lorenceau and Quéré8 demonstrating the spontaneous motion of a droplet deposited onto a wet conical fiber, which possesses a variation in the fiber radius. The motion is driven by the established spatial difference in capillary pressure and the direction is from the thin to the thick part of the fiber. Gravitational effects will be weak for droplets much smaller than the capillary length $L_{c}=\sqrt{\sigma /(\rho g)}$, where $\sigma$ is the surface tension, $\rho$ the liquid density, and $g$ is the gravitational acceleration. The ratio of gravity to surface tension

\footnotetext{
a) Author to whom correspondence should be addressed. Electronic mail: sigurdur.thoroddsen@kaust.edu.sa.
} 
is expressed by the Bond number, $B o=\rho g R^{2} / \sigma$, where $R$ is the droplet radius. In our study, $B o$ is always small and gravity only has effects for the very largest droplets.

The capillary number

$$
C a=\frac{\mu V}{\sigma},
$$

where $\mu$ is the liquid dynamic viscosity and $V$ is the droplet translational velocity, expresses the competition between viscous forces and surface tension. It is also small in our study.

For small values of the $B o$ and $C a$, we expect the droplet shapes to show small deviations from the static shapes determined with use of the Young-Laplace equation, which expresses the jump in pressure across a free surface in terms of $\sigma$ and the principal radii of curvature $R_{1}, R_{2}$, as

$$
\Delta P=\sigma\left(\frac{1}{R_{1}}+\frac{1}{R_{2}}\right)
$$

Carroll ${ }^{18}$ showed that for a stationary droplet that sits on a uniform cylindrical fiber, this pressure jump can be expressed as $\Delta P=2 \sigma /(r+h)$, where $r$ is the local fiber radius and $h$ is the maximal thickness of the droplet, as sketched in Fig. 1(a). However, when a droplet sits on a tapered fiber, the varying radius sets up a spatial gradient of $\Delta P$ along the droplet, as

$$
\left.\frac{d P}{d z}\right|_{\Omega}=-\frac{2 \sigma}{(r+h)^{2}}\left(\frac{d r}{d z}+\frac{d h}{d z}\right)_{\Omega}
$$

where the pressure $P$ inside the droplet is evaluated as a function of its position along the fiber $z$ (as defined in Fig. 1(a)), its maximal thickness $h$, the liquid surface tension $\sigma$, and the local fiber radius $r$. Here $\Omega$ is droplet volume and the subscript indicates that the derivatives are evaluated keeping $\Omega$ constant. According to this pressure gradient, a curvature-driven motion will occur. Following Lorenceau and Quéré, ${ }^{8}$ this pressure gradient can be further simplified for thin vs quasi-spherical drops, by comparing the local fiber radius $r$ to the equivalent droplet radius $R_{0}=(3 \Omega / 4 \pi)^{1 / 3}$, into

$$
\left.\frac{d P}{d z}\right|_{\Omega}=-\frac{2 \sigma \alpha}{\left(r+R_{0}\right)^{2}} \quad \text { for } \quad r<R_{0} ;\left.\quad \frac{d P}{d z}\right|_{\Omega}=-\frac{\sigma \alpha}{r^{2}} \quad \text { for } \quad r>R_{0},
$$

i.e., the two regimes shown in Figs. 1(b) and 1(c), respectively.

Next step is to evaluate the viscous friction, which will counteract the capillary driving force. The nature of the friction is different for thin drops, where it is global occurring along the entire drop, vs near-spherical drops where most of the friction occurs near the two ends, where the drop surface meets with the curvature of the cone, i.e., local friction. ${ }^{8}$

Following Lorenceau and Quéré, ${ }^{8}$ the viscous friction acts mostly within the liquid wedge of angle $\theta$ defined in Fig. 1(a). The no-slip boundary condition causes the stress to increase with the velocity gradient when the layer becomes thinner close to the end of the wedge, i.e., $\sim \mu(d v / d x)$, where $x$ is in the radial direction (Fig. 1(a)). The thickness of the wedge changes as $\theta z$, where $z$ is measured from the tip of the wedge. This gives a local magnitude of the friction $d f \sim \mu V /(\theta z)$, which must be integrated to find the total friction. For drop moving on a dry solid, this integration gives a logarithmic singularity near the tip of the wedge, which must be modeled by a molecular cut-off length. ${ }^{19}$ This integration yields $\mu V r l / \theta$, where the factor $l$ arises from the logarithmic cut-off. ${ }^{8}$ Hoffman ${ }^{19}$ showed $l \simeq 15$ for liquid spreading on a dry surface, while Bico and Quéré ${ }^{20}$ obtained $l \simeq 5$ for liquid spreading on a wet surface, a value we will use herein. The reduction of the viscous friction in the latter case results from the wedge slipping on the pre-wetted film. It remains to determine the wedge angle $\theta$. For a quasi-spherical droplet, $\theta$ is given by Hoffman-Tanner's law, $8,19,21 \theta \sim C a^{1 / 3} \sim(l \mu V / \sigma)^{1 / 3}$, which expresses a local balance between surface tension and viscosity. Substituting this relation into the above expression, we arrive at

$$
f \sim r \sigma^{1 / 3}(\mu l V)^{2 / 3}
$$

for quasi-spherical droplets.

For a thin droplet, the wedge angle becomes global and is simply determined by the geometry, ${ }^{8}$ i.e., $\theta \simeq 2(h-r) / L$, where $L$ is the droplet length as shown in Fig. 1(a). The viscous friction is then 


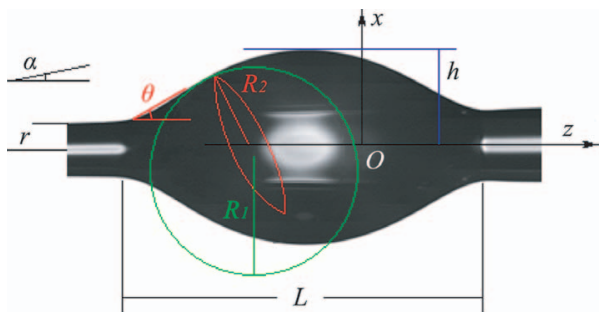

(a)

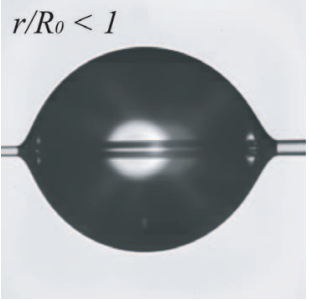

(b)

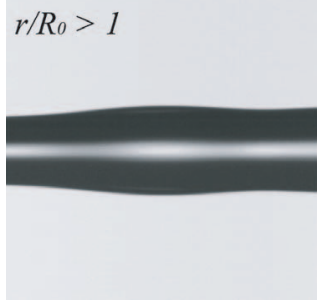

(c)

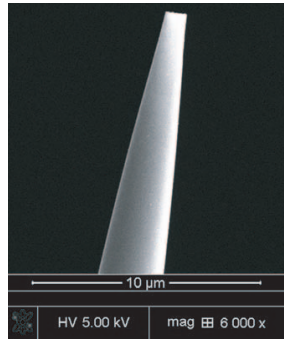

(e)

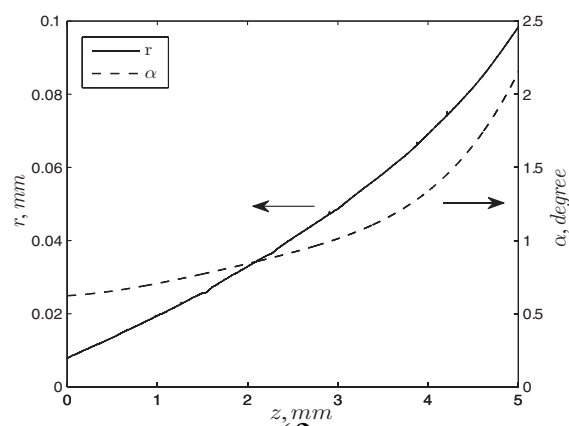

(f)

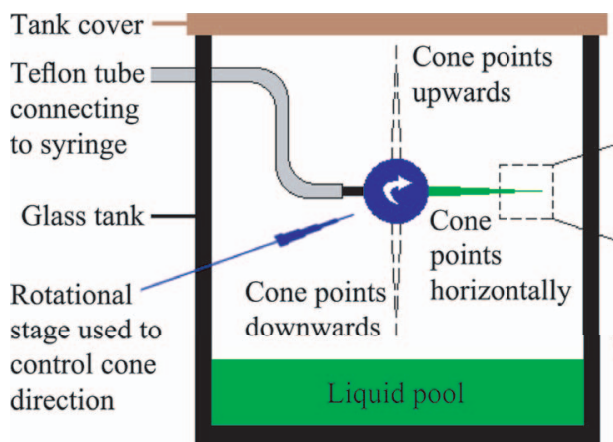

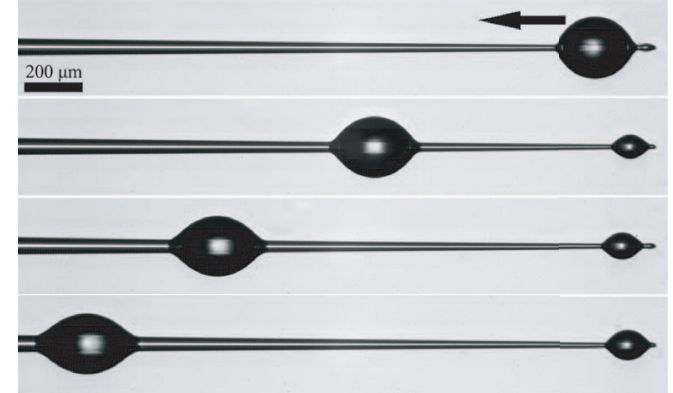

(g)

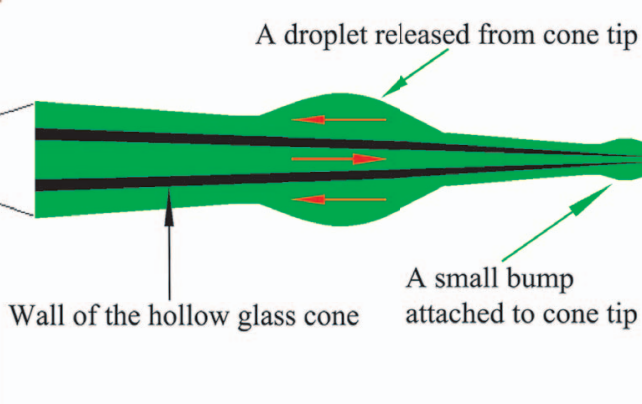

(h)

FIG. 1. (a) Shape of a wetting droplet on a conical fiber with $r$ the local fiber radius and $\alpha$ the semi-angle of the fiber. (b) A droplet (equivalent spherical radius $R_{0}$ ) on a fiber with $R_{0}=175 \mu \mathrm{m}$ and $r=11 \mu \mathrm{m}$. (c) A thin droplet on a fiber with $R_{0}=42 \mu \mathrm{m}$ and $r=66 \mu \mathrm{m}$. (d) Images of a pulled glass capillary tube. Image includes two straight lines to guide the eyes. (e) A scanning electron microscope (SEM) image showing profile and surface smoothness of the pulled cones used in the study. (f) Radius $r$ (left ordinate) and semi-angle $\alpha$ (right ordinate) of the conical tube vs length. (g) Climbing of a $10 \mathrm{cSt}$ silicone oil droplet on a downwards pointing cone. Times relative to first frame are $0,136,272$, and $408 \mathrm{~ms} . R_{0}=220 \mu \mathrm{m}$ and $r_{0}=8 \mu \mathrm{m}$. (h) A schematic showing a cone inside the sealed glass tank. Close-up schematic drawing shows the liquid flow directions inside and outside the cone (red arrows). The wetting layer is much thinner than sketched. 
evaluated as

$$
f \sim \mu l V r \frac{L}{(h-r)} .
$$

The critical transition velocity $V_{c}$ is found by equating these two dissipations rates, which gives

$$
V_{c} \sim \frac{\sigma}{\mu l}\left(\frac{h-r}{L}\right)^{3} .
$$

Accordingly, the dissipation is global if $V>V_{c}$, otherwise it is local.

By equating the viscous friction to the driving force above, we get for thin droplet $\left(r>R_{0}\right)$ subjected to local $\left(V_{\text {local }}<V_{c}\right)$ or global $\left(V_{\text {global }}>V_{c}\right)$ dissipation

$$
V_{\text {local }}^{\text {thin }} \sim \frac{\sigma}{\mu l}\left(\frac{\alpha \Omega}{r^{3}}\right)^{1.5} ; \quad V_{\text {global }}^{\text {thin }} \sim \alpha \frac{\sigma}{\mu l}\left(\frac{h-r}{L}\right) \frac{\Omega}{r^{3}},
$$

whereas for quasi-spherical droplet $\left(r<R_{0}\right)$

$$
V_{\text {local }}^{\text {sphere }} \sim \frac{\sigma}{\mu l}\left[\frac{2 \alpha \Omega}{r\left(r+R_{0}\right)^{2}}\right]^{1.5} ; \quad V_{\text {global }}^{\text {sphere }} \sim 2 \alpha \frac{\sigma}{\mu l}\left(\frac{h-r}{L}\right) \frac{\Omega}{r\left(r+R_{0}\right)^{2}} .
$$

Lorenceau and Quéré ${ }^{8}$ reported good agreement between $V_{\text {global }}^{\text {thin }}$ and their experimental results. However, the use of relatively larger fibers ( $r$ increases from $150 \mu \mathrm{m}$ to $350 \mu \mathrm{m}$, in $3 \mathrm{~cm}$ ) sets a limit on evaluating the climbing of larger droplet in their study, as the capillary length $L_{c}=\sqrt{\sigma /(\rho g)}$ which is around $1.49 \mathrm{~mm}$ for silicone oil can be easily reached in this case. Furthermore, the fastest droplet velocity reported in their study is around $10 \mathrm{~mm} / \mathrm{s}$ for silicone oil of viscosity $\mu=5 \mathrm{mPa}$. As Eqs. (5) and (6) show that a thinner fiber is always preferable for fast droplet motion, which is of interest in potential application in biomedicine or chemistry, this motivated us to evaluate this droplet climbing phenomenon under a much smaller length scale. For comparison of the typical fiber used by Lorenceau and Quéré, ${ }^{8}$ the diameter increased from 300 to $700 \mu \mathrm{m}$ over a length of $3 \mathrm{~cm}$, with an average semi-angle $\alpha \simeq 0.38^{\circ}$. In the present study, the diameter of a typical conical glass tube starts at $\sim 16 \mu \mathrm{m}$ and increases to $\sim 140 \mu \mathrm{m}$ over $4 \mathrm{~mm}$, with an average $\alpha \simeq 0.89^{\circ}$. Our cones are therefore about 10 times smaller.

\section{EXPERIMENTAL SETUP}

Herein we use conical glass tubes produced by heating and pulling glass capillaries (TW100-4, from World Precision Instruments with $1.5 \mathrm{~mm}$ outer diameter and $0.2 \mathrm{~mm}$ wall thickness) with a micropipette puller (P-97 Flaming/Brown type from Sutter Instrument). The capillary breaks into two parts at the end of the pulling with each of them containing a hollow cone with a sharp solid tip. The profile of the pulled tubes can be controlled by varying the heating temperature and pulling velocity. The typical resulting shape is shown in Fig. 1(d1). Here we have produced a cone with a diameter starting at $\sim 2 \mu \mathrm{m}$ at the left of the image and increasing to $\sim 72 \mu \mathrm{m}$, over a length of about $3.2 \mathrm{~mm}$. This gives an average cone semi-angle $\alpha \sim 35^{\circ} / 3200^{\circ} \sim 0.63^{\circ}$. The end of the tube is then hit by a heated wire under a microscope to break off the curved tip and open up the hollow interior, as shown in Fig. 1(d2). The glass capillary heating and pulling processes are highly repeatable, thus a bulk of numerous cones with nearly the same profile can be fabricated.

The glass capillaries are made of borosilicate (Duran ${ }^{\circledR} 8330$ ). The liquids used are all partially or fully wetting on this glass. The measured static contact angles on a flat surface of this glass material are $21^{\circ}$ for water, $4^{\circ}$ for $10 \mathrm{cSt}$ silicon oil, $9^{\circ}$ for $100 \mathrm{cSt}$ silicon oil, $13^{\circ}$ for $1000 \mathrm{cSt}$ silicon oil, and $8^{\circ}$ for perfluorohexane. Methanol fully wets a flat glass substrate.

Typically, glass surfaces have a few nanometer roughness, depending somewhat on their fabrication method. We have also verified that the glass surface remains very smooth after the heating 
and pulling of the tube. This is clear in Fig. 1(e), which shows a scanning electron microscope (SEM) image of a typical pulled cone used in the present experiments. We can see that even close to the sharp tip of the cone, where the local fiber radius $r \simeq 0.5 \mu \mathrm{m}$, the surface roughness is still imperceptible and much smaller as compared to the cone diameter. From this image we can put an upper bound of $50 \mathrm{~nm}$ for this roughness. This is relatively a much smoother surface than that produced by chemical etching of the copper cones used by Lorenceau and Quéré ${ }^{8}$ (Figure 3 of Ref. 8). Furthermore, these small roughnesses do not affect the droplet motions on pre-wetted cones used in these studies.

To accurately measure $r$ and $\alpha$ of the conical tube (as well as the droplet profile), long-distance microscopes were used with Mitutoyo objectives at magnifications of 5, 10, 20, 50, 100, or 200×, which give the corresponding pixel resolutions of $4,2,1,0.4,0.2$, and $0.1 \mu \mathrm{m}$. The measured fiber radius $r$ and the calculated cone semi-angle $\alpha$ are shown as a function of the distance $z$ from the tip in Fig. 1(f). It is shown that the cone has a diverging profile with $\alpha$ varying between 0.011 and 0.038 , within a length of $z=5 \mathrm{~mm}$.

The cone was inserted into a sealed glass tank, which contained a layer of the same liquid (Fig. 1(h)), especially when a volatile liquid was used. This was done to prevent fast evaporation to guarantee wetting of the cone and to minimize thermocapillary effects. As this study focuses only on droplets climbing on wet fibers, a pre-wetting film of the same liquid as the droplet is required on the fiber outer surface. This can be achieved by releasing a few drops immediately before the actual measurement. These early drops will leave a wetting film on the cone.

For most of the experiments, the fiber is kept vertical (except in Fig. 4(b)) with the sharp tip pointing downwards. Gravity therefore works against the motion, but does not break the axisymmetry of the free surface. The rapid surface motions of the droplets require high-speed video at frame rates up to $150 \mathrm{kfps}$ acquired with a Photron SA-5 CMOS video camera. The elongated geometry allows frame rates up to $\sim 50 \mathrm{kfps}$ at resolution of $128 \times 1024$ pixels. Backlighting was accomplished with a $350 \mathrm{~W}$ metal-halide lamp (Sumita Optical Glass, Inc.), which was shone onto a diffuser. The liquids used were water, methanol, perfluorohexane, and silicone oils of various viscosities as listed in Table I.

It is observed that the volume of a methanol droplet is almost constant during the climbing, see Fig. 2(a). This verifies the stability of the thin liquid film which prewets the fiber surface, as well as the effectiveness of using the sealed glass tank to prevent fast evaporation of the volatile liquids. The positions and profiles of the droplets during climbing are measured from the recorded high-speed video frames, using a thresholding program implemented in MATLAB. We calculate the droplet shape factors $h$ and $L$, and integrate the axisymmetric free surface and compare with the dry cone shape to find the droplet volume $\Omega$. By tracking the location of the maximum droplet thickness $h$, the droplet velocity $V$ can be deduced. Droplet velocity was measured for a range of droplet sizes and viscosities. The duration of the applied pressure determines the amount of fluid forced out of the tube and therefore the size of the individual droplets, which subsequently travel up the cone.

TABLE I. Properties of the different liquids used in the experiments. Room temperature was kept at $21^{\circ} \mathrm{C}$.

\begin{tabular}{lcccc}
\hline \hline Liquid & $\begin{array}{c}\text { Density } \\
\rho\left(\mathrm{kg} / \mathrm{m}^{3}\right)\end{array}$ & $\begin{array}{c}\text { Viscosity } \\
\mu(\mathrm{cP})\end{array}$ & $\begin{array}{c}\text { Surface tension } \\
\sigma(\mathrm{N} / \mathrm{m})\end{array}$ & $\begin{array}{c}\text { Capillary length } \\
L_{c}(\mathrm{~mm})\end{array}$ \\
\hline Water & 998 & 1.001 & 0.0721 & 2.72 \\
Methanol & 792 & 0.571 & 0.0225 & 1.70 \\
Silicon oil 10 & 930 & 9.30 & 0.0201 & 1.49 \\
Silicon oil 100 & 965 & 96.5 & 0.0209 & 1.49 \\
Silicon oil 500 & 970 & 485 & 0.0211 & 1.49 \\
Silicon oil 1000 & 970 & 970 & 0.0212 & 1.49 \\
Perfluorohexane & 1710 & 1.104 & 0.0119 & 0.843 \\
\hline \hline
\end{tabular}




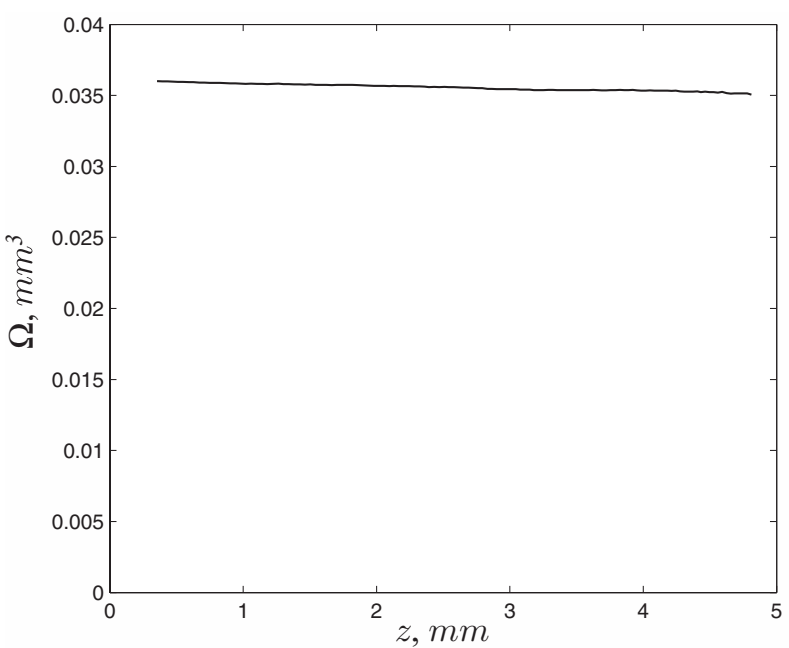

(a)

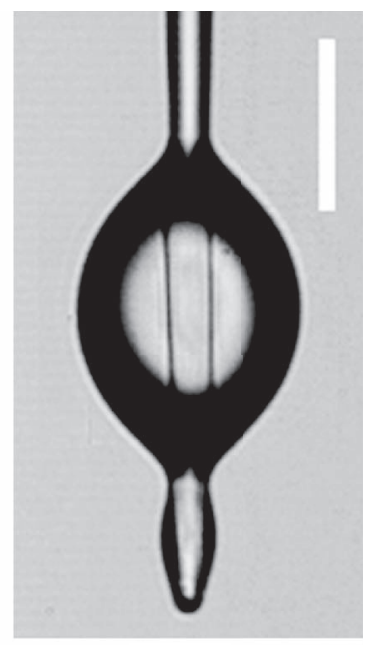

(b)

FIG. 2. (a) The volume of a methanol droplet as it climbs up along a conical fiber that is inserted into a sealed glass tank, which contains a layer of methanol at its bottom. $R_{0}=203 \mu \mathrm{m}$ and $r_{0}=15 \mu \mathrm{m}$. (b) A droplet stuck at the end of the cone. $R_{0}=17 \mu \mathrm{m}$ and the tip $r_{0}=2 \mu \mathrm{m}$. The scale bar is $20 \mu \mathrm{m}$.

\section{RESULTS}

\section{A. Raw data}

Prior to the climbing, a droplet will be stuck at the end of the cone with its lower end being connected with another even smaller liquid bump, as shown in Fig. 2(b) (see Movie S1 in the supplementary material ${ }^{22}$ for details). The thickness of this connecting film is measured to be only $\sim 1-2 \mu \mathrm{m}$. The droplet grows in size as the flow entering it, followed by a sudden release once the squeezed inflow is stopped. This detachment can produce a transient oscillation of high frequency (Movie $\mathrm{S} 1$ in the supplementary material ${ }^{22}$ shows a release oscillation with a frequency of $14 \mathrm{kHz}$ ). Equations (5) and (6) tell us that the speed of the droplets depends primarily on the liquid physical parameters of surface tension and viscosity, as well as on the droplet volume, droplet aspect ratio, and on the cone semi-angle. So, first, Fig. 3(a) shows results for the velocity of water droplets of different volumes, but moving up the same conical tube. As compared to the results reported by Lorenceau and Quéré ${ }^{8}$ where droplets were always decelerated, it is interesting that here the larger droplets are shown to accelerate initially and reach a maximum velocity subsequently decelerating. As the droplet volume is reduced, the maxima move to smaller cone radii. Only for the relatively small droplet $\left(R_{0}<75 \mu \mathrm{m}\right)$, the droplets do decelerate from the very beginning. The largest observed velocity is about a quarter of a meter per second. Figures 3(b)-3(f) show results for the velocity of methanol, perfluorohexane, and silicone oil droplets of different viscosities. Similar trends are observed for all of the liquids, but it is noted that all the velocity magnitudes decrease greatly with increasing viscosity.

It should be noted that the results shown in Figs. 3(a)-3(f) were obtained by using relatively thin cones with radius $r$ ranges from 8 to $80 \mu \mathrm{m}$ during droplet climbing. So the results should be considered as for quasi-spherical droplets $\left(r<R_{0}\right)$. To verify Eq. (5) for thin droplets $\left(r>R_{0}\right)$, the fiber was broken off a further distance from the tip to give larger opening $r_{0} \sim 33 \mu \mathrm{m}$. The corresponding results when $r>R_{0}$ are shown in Fig. 4(a). Movies S2 and S3 in the supplementary material show the climbing of droplets with different sizes and viscosities, respectively. ${ }^{22}$

\section{B. Dynamics of the motion}

As mentioned earlier, the use of large magnifications makes it possible for us to accurately measure the droplet maximal thickness $h$ and length $L$ during climbing. Droplet aspect ratio 


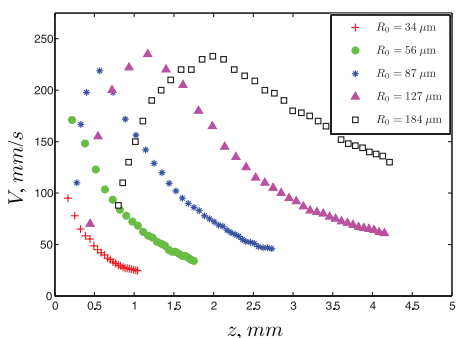

(a)

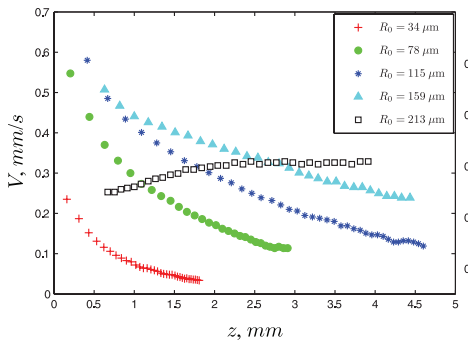

(d)

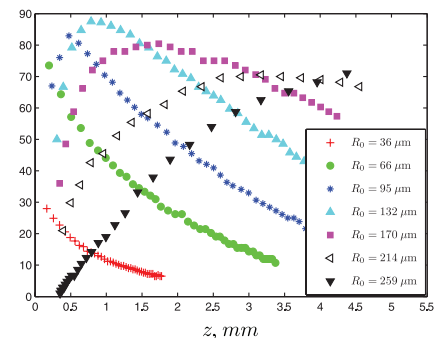

(b)

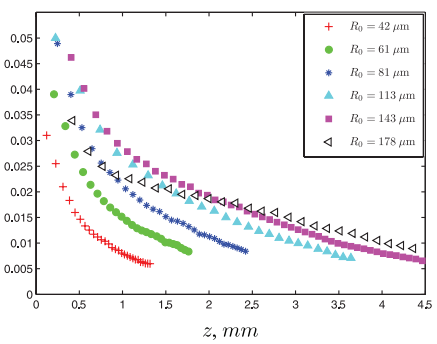

(e)

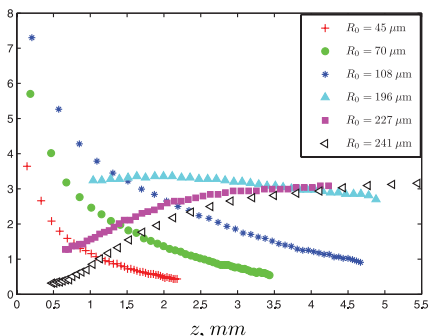

(c)

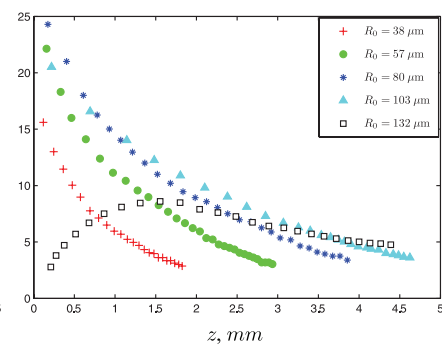

(f)

FIG. 3. Speed of (a) water, (b) methanol, (c) $10 \mathrm{cSt}$ silicone oil, (d) $100 \mathrm{cSt}$ silicone oil, (e) $1000 \mathrm{cSt}$ silicone oil, and (f) perfluorohexane droplets along a conical fiber for various droplet volumes. Cone tips pointed downwards. Cone-tip size $r_{0}=8 \mu \mathrm{m}$.

$(h-r) / L$ and critical velocity $V_{c}$ defined in Eq. (4) then can be calculated with time. It is found that for all the three thin droplets shown in Fig. 4(a), the calculated $V_{c}$ is always lower than the real droplet velocity $V$, implying that the viscous dissipation should be always considered as global. This is reasonable as a thin droplet is always stretched to resemble a global macroscopic wedge, as shown in Fig. 1(c).

Following Lorenceau and Quéré ${ }^{8}$ we first investigate the relationship between droplet velocity and the quantity $\alpha[(h-r) / L] / r^{3}$. As shown in Fig. 5(a), for thin droplets with a fixed volume, droplet velocity is proportional to the quantity $\alpha[(h-r) / L] / r^{3}$, with the proportionality constant changing with droplet size. This agrees well with the results of Lorenceau and Quéré ${ }^{8}$ and implies that the deceleration along the climbing is due to a decrease in driving force as well as an increase in the friction force. Figure 5(b) presents the droplet velocity $V_{\text {global }}^{\text {thin }}$ evaluated by Eq. (5) for 3 different droplet volumes. It is clearly shown that after normalizing by droplet volume $\Omega$ and capillary viscous

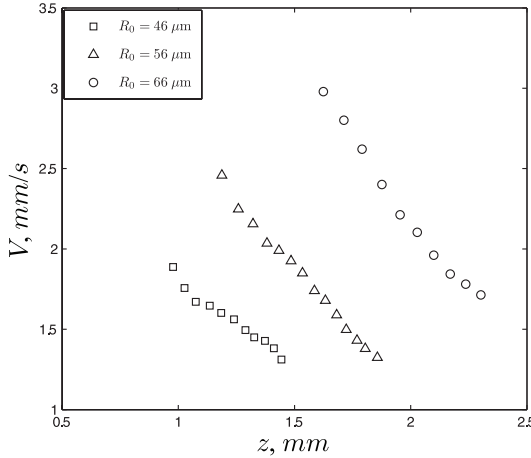

(a)

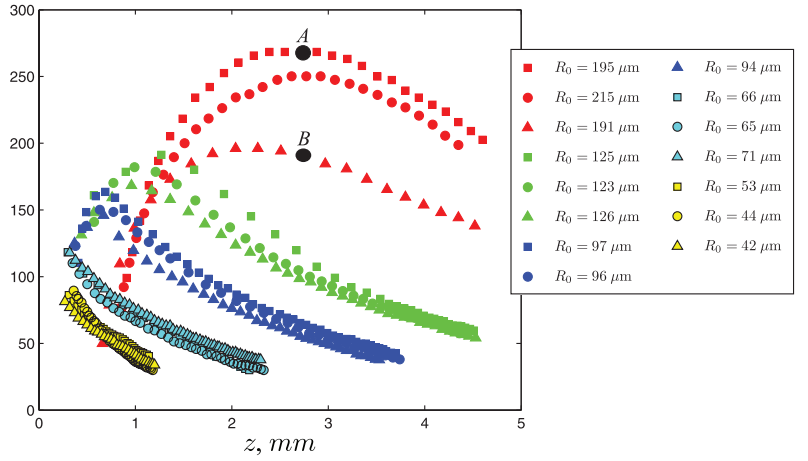

(b)

FIG. 4. (a) Speed of small water droplets of three different volumes during climbing. Data points collected when the local fiber radius $r>R_{0}$. Cone tip pointed downwards. Cone-tip $r_{0}=33 \mu \mathrm{m}$. (b) The effects of the cone orientation on the speeds of water droplets moving along a conical fiber. Cone tip pointed upwards $(\square)$, horizontally $(\bigcirc)$, and downwards $(\triangle)$ for $r_{0}$ $=9 \mu \mathrm{m}$. 


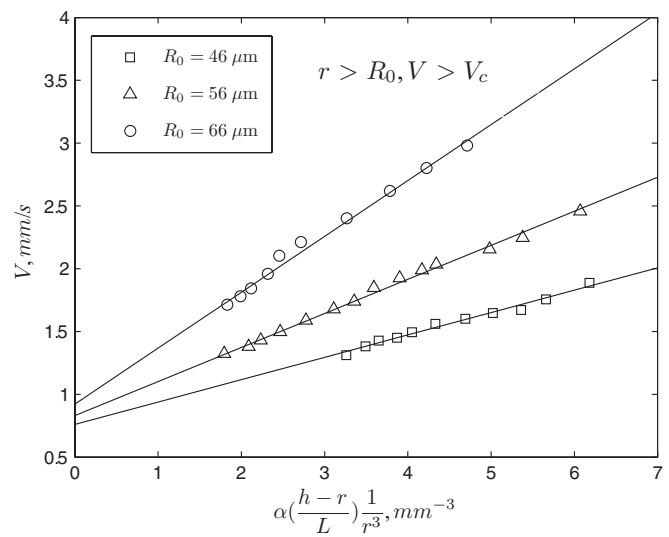

(a)

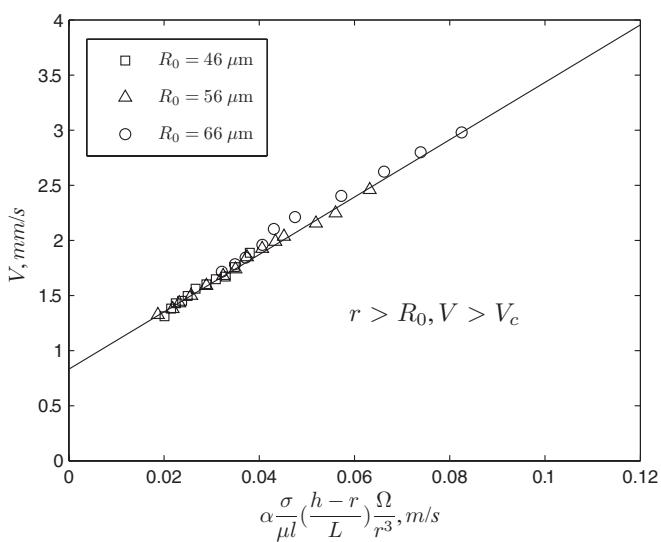

(b)

FIG. 5. Droplet speed as a function of the quantities (a) $\alpha[(h-r) / L]\left(1 / r^{3}\right)$ and (b) $(\alpha \sigma /(\mu l))[(h-r) / L]\left(\Omega / r^{3}\right)$ for water droplets of different volumes moving along the same conical fiber. $V>V_{c} \cdot r_{0}=33 \mu \mathrm{m}$. Cone tip pointed downwards. Data points collected when the local fiber radius $r>R_{0}$.

velocity, the different curves for thin droplets collapse onto each other (the prefactor of the straight line is around 0.026) showing very good agreement with Eq. (5).

For quasi-spherical droplets $\left(r<R_{0}\right), V_{\text {local }}^{\text {sphere }}$ and $V_{\text {global }}^{\text {sphere }}$ in Eq. (6) will be investigated for $V<V_{c}$ (local dissipation) and $V>V_{c}$ (global dissipation), respectively. Figure 6(a) presents the droplet velocity as a function of the quantity that is defined by Eq. (6), when $V<V_{c}$. It is clearly shown that different curves collapse onto each other and fall onto a straight line with a prefactor of 0.060 , except a large deviation during the acceleration part of relatively large droplets. It should be noted here that the variations in $\alpha$ have been included into the $x$-axis, so the diverging cone profile (shown in Fig. 1(f)) should not be the reason for this deviation. This will be further discussed in Sec. III E. Figure 6(b) presents the droplet velocity as a function of the quantity that is defined by Eq. (6) when $V>V_{c}$. Again, different curves collapse onto each other (the prefactor of the straight line is around 0.164) showing good agreement with Eq. (6).

\section{Effects of viscosity}

Figure 3 showed that droplet speed is generally decreased with increasing viscosity. This is clearly revealed in Fig. 7(a), where we plot the maximum climbing speed for each liquid showing

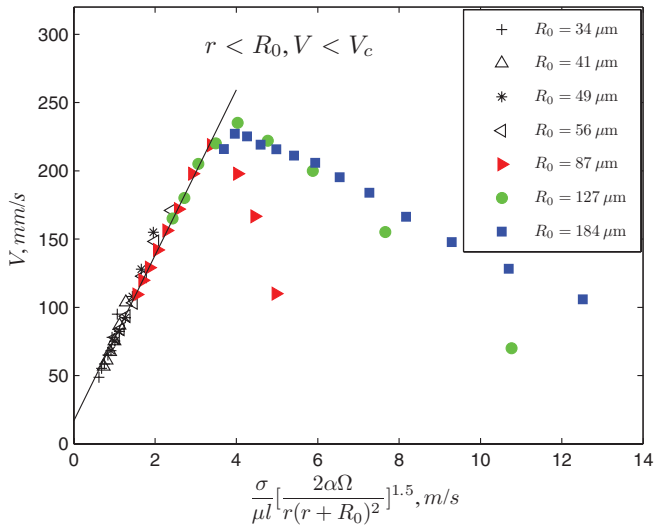

(a)

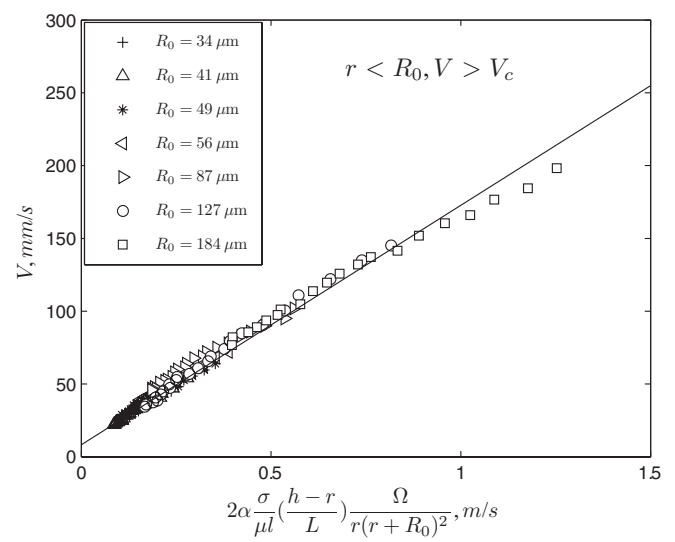

(b)

FIG. 6. Droplet speed defined by (a) $V_{\text {local }}^{\text {sphere }}$ and (b) $V_{\text {global }}^{\text {sphere }}$, in Eq. (6), for water droplets of different volumes moving along the same conical fiber. Cone tip pointed downwards. $r_{0}=8 \mu \mathrm{m}$. 


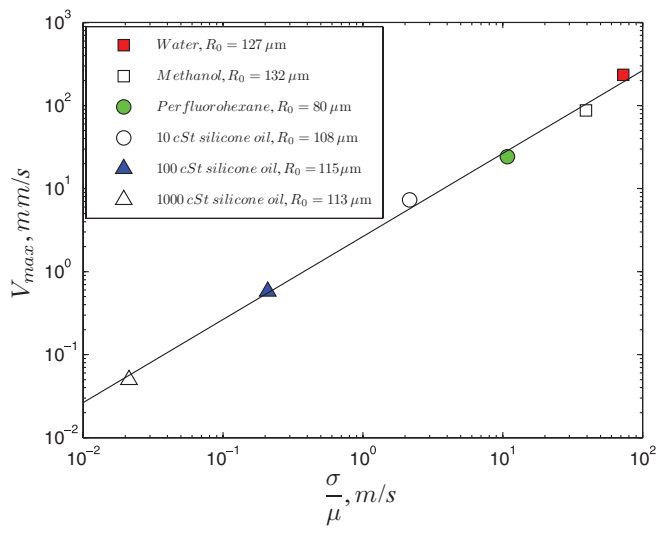

(a)

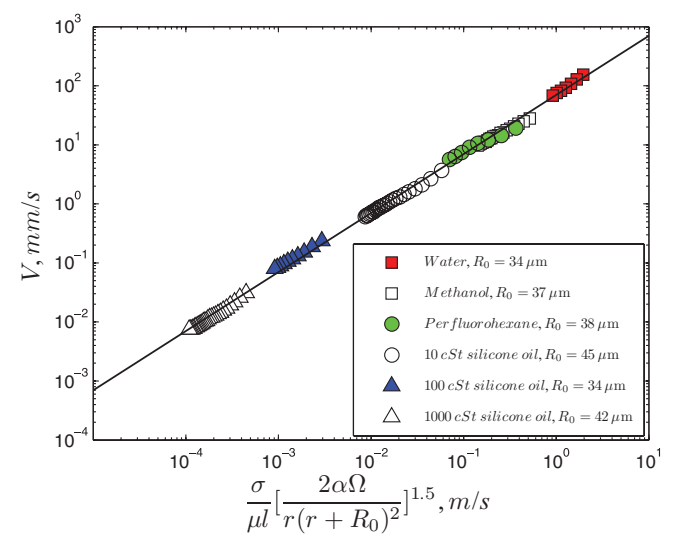

(b)

FIG. 7. (a) Maximum droplet speed as a function of the capillary-viscous velocity of the various liquids listed in Table I. The trendline has a slope of 1. (b) Measured droplet speed vs $V_{\text {local }}^{\text {sphere }}$ of Eq. (6), for droplets of different liquids moving along identical conical fibers, with $r_{0}=8 \mu \mathrm{m}$. The trendline has a slope of 1 . All cone tips pointed downwards.

linear increase as a function of the capillary-viscous velocity $\sigma / \mu$ on a $\log / \log$ scale with a slope of 1 , a typical feature in a viscous dominated flow. This is further suggested by the highest Reynolds numbers $R e=\rho V 2 R_{0} / \mu$ in the experiment, which are around 60 for water, 35 for methanol, and even smaller for the silicone oils. Thereby, after a short acceleration phase for the smallest viscosities, the droplets should reach a balance between the capillary driving force and viscous friction. The results are expected but still encouraging to see it work over 3.5 orders of magnitude.

Figure 7(b) presents droplet velocity $V_{\text {local }}^{\text {sphere }}$ defined by Eq. (6) for different liquids. Experimental results show excellent agreement with Eq. (6) as all the curves fall onto the same straight line which has a slope of 1 .

\section{Effects of gravity}

Gravitational effects have been ignored in all the previous equations, which is expected to be valid for the smaller droplets or for horizontal fibers. To evaluate this effect directly, experiments have been carried out with water droplets traveling along the same fiber, but with the cone tip pointed downwards, horizontally, or upwards. The results are presented in Fig. 4(b) with each colour marking droplets of similar sizes, but traveling in the three different directions.

For the smallest droplets, the gravitational effects are minimal. However, for relatively big droplets $\left(R_{0}>90 \mu \mathrm{m}\right)$, the ones moving along the gravitational direction (when the cone tip points upwards) have the highest velocities (denoted here by $V_{1}$ ); the ones moving against gravity (when the cone tip points downwards) have the lowest velocities (denoted by $V_{3}$ ); and the ones moving horizontally have intermediate velocities (denoted by $V_{2}$ ). The largest differences between $V_{1}$ and $V_{3}$ are about $45 \%, 24 \%$, and $18 \%$ for $R_{0} \sim 195 \mu \mathrm{m}, 125 \mu \mathrm{m}$, and $95 \mu \mathrm{m}$, respectively. This difference becomes insignificant for the smallest droplets, and the curves for $V_{1}, V_{2}$, and $V_{3}$ almost overlap each other. Keep in mind that this $D_{0}=2 R_{0}=180 \mu \mathrm{m}$ is 15 times smaller than the capillary length for water and would thereby have little effect on the sphericity of a drop. However, the driving force arises from the gradient of the capillary pressure and is a weaker force. The Bond numbers are always low in our study in the range from $2 \mathrm{E}-04$ to 0.026 .

For the largest droplets we can use this gravity-affected velocity difference to obtain two independent equations to find the strength of the capillary driving force and the resistive viscous stress. Newton's second law gives

$$
m a=\sum F=F_{\sigma}-F_{\mu} \pm \rho g \Omega,
$$

where $F_{\sigma}$ is the capillary pressure driving force, $F_{\mu}$ is the viscous friction, and the last term representing gravity for upwards $(+)$ and downwards $(-)$ facing cone. For quasi-spherical droplet 
and local dissipation, we have $F_{\sigma}=2 C_{\sigma} \sigma \alpha \Omega /\left(r+R_{0}\right)^{2}$ and $F_{\mu}=C_{\mu} r \sigma^{1 / 3}(\mu l V)^{2 / 3}$, where $C_{\sigma}$ and $C_{\mu}$ are unknown proportionality constants. By looking at the maximum velocity where the acceleration is negligible, we can use $\sum F=0$, see points $A$ and $B$ marked in Fig. 4(b). By comparing the real up and down experimental data, the constants $C_{\sigma}$ and $C_{\mu}$ are calculated to be 2.04 and 13.2, respectively. Accordingly, the prefactor of $V_{\text {local }}^{\text {sphere }}$, in Eq. (6), becomes $\left(C_{\sigma} / C_{\mu}\right)^{1.5}$ $=0.061$, which is in good agreement with the value 0.060 , i.e., the prefactor that was obtained from the linear fit in Fig. 6(a).

\section{E. Why local maximum velocity is produced for big droplets}

From Eq. (3) we see that the driving pressure gradient for a climbing droplet on a conical fiber should decrease with $r$, if $\alpha$ is constant. Even though the fibers used in this study have a weakly diverging profile, such that $\alpha$ increases slightly with $z$, we do not expect $d \alpha / d z$ to have significant effects locally over the length of the drop, but only over the length of the fiber. Therefore, the driving force $\left.(d P / d z)\right|_{\Omega} \sim-\sigma \alpha / r^{2}$ will monotonically decrease with $z$, as fiber radius $r \sim \alpha z$. This is consistent with our results as thin droplets always slow down during climbing. For quasi-spherical droplets, $\left.(d P / d z)\right|_{\Omega}=-2 \sigma \alpha /\left(r+R_{0}\right)^{2}$ is showing that the pressure gradient along $z$ is not only a function of $\alpha$ and $r$, but also of $R_{0}$. It can be imagined that over a short time following the release of droplet from the cone tip (i.e., when $z$, as well as $r$, is much smaller than $R_{0}$ ), this pressure gradient will increase with $z$ for droplets with relatively big $R_{0}$, as the cone has a diverging profile. Figure 8(a) shows the calculated net driving force $F=\Omega\left[2 C_{\sigma} \sigma \alpha /\left(r+R_{0}\right)^{2}-\rho g\right]$ for water droplets traveling up against gravity, with geometric parameters $\alpha$ and $r$ taken from the cone in Fig. 1(f). For accuracy, gravitational effects have also been taken into account here. It is clearly shown that the net driving force for relatively small droplets (when we still have $R_{0}>r$ ) decreases monotonically with $z$, while it increases slightly once $R_{0}$ exceeds $125 \mu \mathrm{m}$. However, for local dissipation, by balancing this net driving force with the viscous friction defined by $F_{\mu}=C_{\mu} r \sigma^{1 / 3}(\mu l V)^{2 / 3}$, the droplet velocity is expressed as

$$
V=\frac{1}{\mu l \sqrt{\sigma}}\left[\frac{2 C_{\sigma} \sigma \alpha \Omega}{C_{\mu} r\left(r+R_{0}\right)^{2}}-\frac{\rho g \Omega}{C_{\mu} r}\right]^{1.5} .
$$

As shown in Fig. 8(b), the predicted velocities decrease monotonically with $z$. Through Figs. 8(a) and 8 (b), it is shown that although the diverging cone profile produces an increase in the net driving force for a climbing droplet with a very large $R_{0}$, the velocities resulting from the force balance still keep decreasing with distance along the cone.

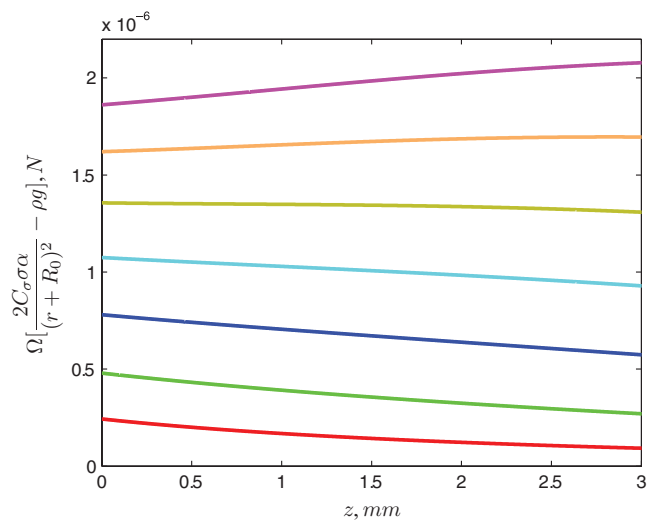

(a)

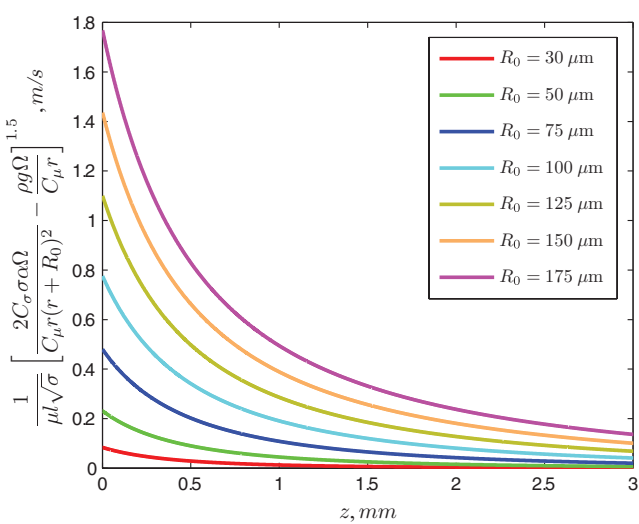

(b)

FIG. 8. For quasi-spherical droplet and local dissipation, (a) calculated net driving force for different droplet volumes and (b) calculated velocity by force balance between capillary, viscous stress, and gravity, $V=$ $(1 / \mu l \sqrt{\sigma})\left[2 C_{\sigma} \sigma \alpha \Omega /\left(C_{\mu} r\left(r+R_{0}\right)^{2}\right)-\rho g \Omega /\left(C_{\mu} r\right)\right]^{1.5}$, for different droplet volumes. Geometric parameters $\alpha$ and $r$ taken from the nozzle shown in Fig. 1(f). Cone tips point downwards. 


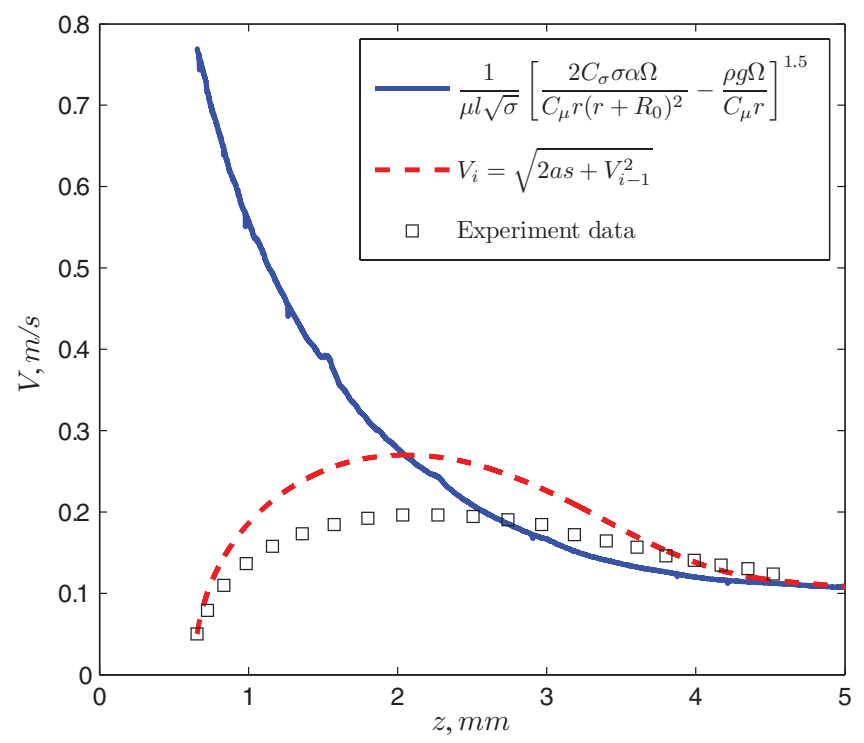

(a)

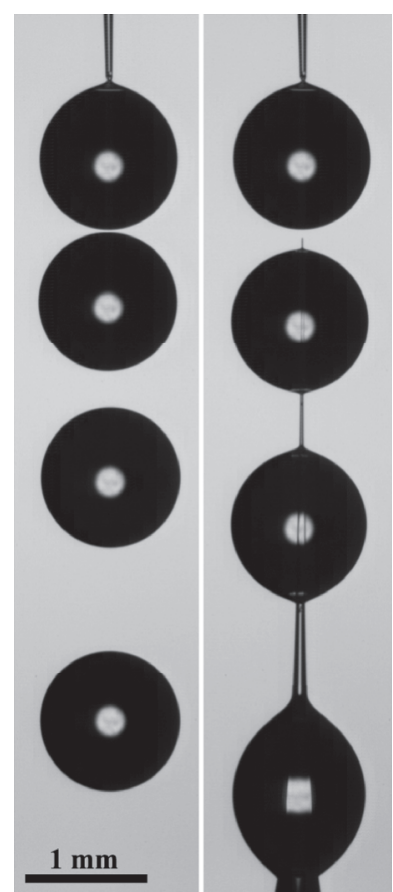

(b)

FIG. 9. (a) Comparison of the velocity calculated by local force balance (solid blue line), the velocity by numerically integrating Eq. (6), i.e., by $V_{i}=\sqrt{2 a s+V_{i-1}^{2}}$ (red line), and the experiment data ( $\square$ black squares), for $R_{0}=191 \mu \mathrm{m}$. Geometric parameters $\alpha$ and $r$ are taken from the nozzle shown in Fig. 1(f). Cone tip pointed downwards. (b) Stroboscopic collage for a free-falling droplet in air (left) and onto an upwards-pointing conical fiber (right). Before falling (right), there was a $0.7 \mathrm{~mm}$ distance from the center of the hanging droplet to the tip of the conical fiber ( $50 \mu \mathrm{m}$ gap from bottom of the hanging droplet to the cone tip). Both droplets have a diameter of $1.3 \mathrm{~mm}$. The snapshots shown are $0,16.3,24.5$, and 32.6 $\mathrm{ms}$ relative to the top image, as the droplets detach from the nozzle.

Based on this, we conclude that the peaks in velocity observed in Fig. 3 for the larger droplets are not due to the diverging profile of the cones or an optimal droplet shape, but rather due to inertia and a protracted acceleration phase. Figure 9(a) investigates this assertion by comparing the measured velocities after the droplet detaches from the tip to the velocity predicted by a local static force balance. The blue line represents calculated droplet velocity based on the local force balance ignoring acceleration, whereas the red line includes inertia and calculates droplet velocity based on $F=m a$, with an initial velocity taken from the first measured value of $0.05 \mathrm{~m} / \mathrm{s}$. The droplet trajectory is found by numerically integrating Eq. (7). Figure 9(a) clearly shows that the accelerated motion theory gives a fairly good estimation for droplet motion, thus verifying that the initial droplet climbing is in an acceleration phase.

Furthermore, the characteristic capillary-inertial time scale for the current system can be estimated approximately as $\tau \sim \sqrt{\rho R_{0}\left(r+R_{0}\right)^{2} /(2 \sigma \alpha)}$. For a water droplet with $R_{0}=30 \mu \mathrm{m}$, this equation gives $\tau \sim 170 \mu \mathrm{s}$. During this short a time-scale, the droplet will travel $\sim 170 \mu \mathrm{s} \times 100$ $\mathrm{mm} / \mathrm{s}=17 \mu \mathrm{m}$ (velocity evaluated from Fig. 3(a)), which is a relatively small distance. Thus, acceleration effects can be neglected and the climbing velocity can be evaluated by balancing the viscous friction with the driving force, just as shown in Eqs. (5) and (6). While for a larger water droplet with $R_{0}=175 \mu \mathrm{m}$, it will travel $\tau V \sim 1900 \mu \mathrm{s} \times 150 \mathrm{~mm} / \mathrm{s}=0.285 \mathrm{~mm}$, a relatively large value in this phenomenon, which implies that the initial climbing should be considered as being in an acceleration phase. For a $150 \mu \mathrm{m}$ radius droplet of water, methanol, silicone oils of $10 \mathrm{cSt}, 100 \mathrm{cSt}$, $1000 \mathrm{cSt}$, and perfluorohexane, the calculated values of $\tau$ are around 1.7, 2.7, 3.1, 3.2, 3.2, and 5.5 $\mathrm{ms}$, respectively. Despite being of the same order, in combination with the averaging velocity for each liquid, the distances a droplet travels during these time scales are around 0.19, 0.12, 0.009, 
9E-04, 9E-05, and $0.05 \mathrm{~mm}$, respectively. Thus, we conclude that the initial climbing of $150 \mu \mathrm{m}$ radius water, methanol, or perfluorohexane droplets should occur in an acceleration phase, while for others it should not. This is in good agreement with the results shown in the various panels of Fig. 3.

Another way of supporting this assertion is to impale a droplet onto the tip of a conical fiber to change the initial velocity of the drop, thus reducing the duration of the acceleration phase. Figure 9(b) shows one such attempt where we compare the trajectory of a free-falling droplet in air to a drop impacting at low velocity onto a upwards-pointing conical fiber. The impacting one accelerates faster than the free-falling one. These experiments had problem with dewetting of the fiber between drops and did not give conclusive results.

\section{CONCLUSIONS}

In summary, we have performed an experimental study of the motion of a micro-droplet along a wet conical fiber. The liquid is fed through the glass cone, accumulating at the fiber tip to form droplets, followed by a sudden release once the squeezed inflow is stopped. The detachment of droplet from the fiber tip can produce a transient oscillation at a frequency over $10 \mathrm{kHz}$. The detached droplet is then driven towards the wider side of the cone, by the gradient in capillary pressure, resulting from the varying radius of the cone.

The surface shapes of the droplets are in all cases close to the static shapes determined by the Young-Laplace equation. This is supported by the measured values of the capillary numbers, where $\mathrm{Ca}$ is always smaller than 0.0034 , as can be deduced from Figure 7(a). The Bond number is also low (smaller than 0.026), which allows us to look at more spherical droplets than in previous studies.

By using a wide range of liquid properties and droplet sizes, we have not only verified the dimensional relationships for the droplet velocities, which were presented in the Introduction section, but also determined their prefactors, as shown below. Keep in mind that we have retained the empirical integration constant $l=5$, based on the values from Lorenceau and Quéré 8 for wet fibers. For thin droplets, we find that the motion is always resisted by global viscous dissipation and the climbing velocity can be determined by Eq. (5), i.e.,

$$
V_{\text {global }}^{\text {thin }}=0.026 \frac{\alpha \sigma}{\mu l}\left(\frac{h-r}{L}\right) \frac{\Omega}{r^{3}},
$$

where the prefactor is determined from Fig. 5(b).

On the other hand, for a quasi-spherical droplet, the climbing can be divided into motions resisted by either local dissipation $\left(V<V_{c}\right)$ or by global dissipation $\left(V>V_{c}\right)$. The climbing velocities can thereby be determined by Eq. (6), i.e.,

$$
\begin{array}{rlrl}
V_{\text {local }}^{\text {sphere }} & =0.061 \frac{\sigma}{\mu l}\left[\frac{2 \alpha \Omega}{r\left(r+R_{0}\right)^{2}}\right]^{1.5}, & V<V_{c}, \\
V_{\text {global }}^{\text {sphere }}=0.33 \frac{\alpha \sigma}{\mu l}\left(\frac{h-r}{L}\right) \frac{\Omega}{r\left(r+R_{0}\right)^{2}}, & V>V_{c},
\end{array}
$$

where the prefactors have been determined from Fig. 6.

Overall, we observe that the maximum droplet climbing velocity for each liquid is generally proportional to the capillary-viscous velocity, a typical feature in a viscous dominated flow. We observe this over 3.5 orders of magnitude in $\sigma / \mu$.

However, for the lowest viscosity liquids we find that the largest drops are prevented from reaching their fastest speeds by inertia. In other words, the initial acceleration takes so long that the drop has traveled significant distance up the cone before viscosity limits its speed. This is clearly demonstrated in Figure 6(a), where the velocities start out (on the right side of the graph) far below the velocities on the theoretical curve. One can therefore envision reaching higher velocities by changing the initial conditions by impacting the drop onto the cone, as was attempted in Figure 9(b). The significance of inertia is further supported by the largest Reynolds numbers we can reach, which for water $R e \simeq 60$. 
By contrast, Lorenceau and Quéré ${ }^{8}$ used more viscous liquids with $R e<1$ and therefore always show a monotonically decreasing velocity with distance traveled up the cone.

By using fibers one order of magnitude smaller than previously tested, ${ }^{8}$ in combination with lower viscosity liquids, we have measured 30 times larger droplet velocities. If mercury could wet a conical surface, our results would predict velocities in excess of $10 \mathrm{~m} / \mathrm{s}$ for a $10 \mu \mathrm{m}$ mercury droplet on a submicron fiber, entering a regime beyond current experimental capabilities.

${ }^{1}$ A. R. Parker and C. R. Lawrence, "Water capture by a desert beetle," Nature (London) 414, 33 (2001).

${ }^{2}$ P. J. Bentley and W. F. C. Blumer, "Uptake of water by the lizard, Moloch horridus," Nature (London) 194, 699 (1962).

${ }^{3}$ Y. Zheng, H. Bai, Z. Huang, X. Tian, F.-Q. Nie, Y. Zhao, J. Zhai, and L. Jiang, "Directional water collection on wetted spider silk," Nature (London) 463, 640 (2010).

${ }^{4}$ B. Selva, V. Miralles, I. Cantat and M.-C. Jullien, "Thermocapillary actuation by optimized resistor pattern: Bubbles and droplets displacing, switching and trapping," Lab Chip 10, 1835 (2010).

${ }^{5}$ P. D. Patel, E. S. G. Shaqfeh, J. E. Butler, V. Cristini, J. Blawzdziewicz, and M. Loewenberg, "Drop breakup in the flow through fixed fiber beds: An experimental and computational investigation," Phys. Fluids 15, 1146 (2003).

${ }^{6}$ D. Quéré, "Fluid coating on a fiber," Annu. Rev. Fluid Mech. 31, 347 (1999).

${ }^{7}$ J.-L. Liu, R. Xia, B.-W. Li, and X.-Q. Feng, "Directional motion of droplets in a conical tube or on a conical fibre," Chin. Phys. Lett. 24, 3210 (2007).

${ }^{8}$ É. Lorenceau and D. Quéré, “Drops on a conical wire,” J. Fluid Mech. 510, 29 (2004).

${ }^{9}$ J. Bico, and D. Quéré, "Self-propelling slugs," J. Fluid Mech. 467, 101 (2002).

${ }^{10}$ M. M. Weislogel, "Steady spontaneous capillary flow in partially coated tubes," AIChE J. 43, 645 (1997).

${ }^{11}$ A. Mazouchi and G. M. Homsy, "Thermocapillary migration of long bubbles in cylindrical capillary tubes," Phys. Fluids 12, $542(2000)$.

${ }^{12}$ A. A. Darhuber, J. P. Valentino, J. M. Davis, S. M. Troian, and S. Wagner, "Microfluidic actuation by modulation of surface stresses," Appl. Phys. Lett. 82, 657 (2003).

${ }^{13}$ Z. J. Jiao, X. Y. Huang, N. T. Nguyen, and P. Abgrall, "Thermocapillary actuation of droplet in a planar microchannel," Microfluid. Nanofluid. 5, 205 (2008).

${ }^{14}$ M. G. Pollack, R. B. Fair, and A. D. Shenderov, "Electrowetting-based actuation of liquid droplets for microfluidic applications," Appl. Phys. Lett. 77, 1725 (2000).

${ }^{15}$ J. Bico and D. Quéré, "Liquid trains in a tube," Europhys. Lett. 51, 546 (2000).

${ }^{16}$ Y. Sumino, N. Magome, T. Hamada, and K. Yoshikawa, "Self-running droplet: Emergence of regular motion from nonequilibrium noise," Phys. Rev. Lett. 94, 068301 (2005).

${ }^{17}$ K. Ichimura, S.-K. Oh, and M. Nakagawa, "Light-driven motion of liquids on a photoresponsive surface," Science $\mathbf{2 8 8}$, 1624 (2000)

${ }^{18}$ B. J. Carroll, "The accurate measurement of contact angle, phase contact areas, drop volume, and Laplace excess pressure in drop on fiber system," J. Colloid Interface Sci. 57, 488 (1976).

${ }^{19}$ R. Hoffman, “A study of the advancing interface,” J. Colloid Interface Sci. 50, 228 (1975).

${ }^{20}$ J. Bico and D. Quéré, "Falling slugs," J. Colloid Interface Sci. 243, 262 (2001).

${ }^{21}$ L. H. Tanner, "The spreading of silicone oil drops on horizontal surfaces," J. Phys. D 12, 1473 (1979).

${ }^{22}$ See supplementary material at http://dx.doi.org/10.1063/1.4805068 for Movie S1 showing the detachment of a methanol droplet from a cone tip. Movies S2 and S3 show the climbing of droplets with different sizes and viscosities, respectively. 